\title{
Pulsed electromagnetic fields alleviate streptozotocin-induced diabetic muscle atrophy
}

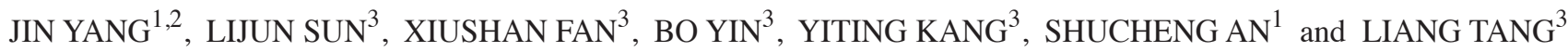 \\ ${ }^{1}$ College of Life Sciences, Shaanxi Normal University, Xi'an, Shaanxi 710062; \\ ${ }^{2}$ Department of Physical Education, Xi'an University of Posts and Telecommunications, Xi'an, Shaanxi 710121; \\ ${ }^{3}$ Institute of Sports Biology, Shaanxi Normal University, Xi'an, Shaanxi 710119, P.R. China
}

Received December 13, 2017; Accepted May 15, 2018

DOI: $10.3892 / \mathrm{mmr} .2018 .9067$

\begin{abstract}
Diabetic muscle atrophy causes a reduction of skeletal muscle size and strength, which affects normal daily activities. However, pulsed electromagnetic fields (PEMFs) can retard the atrophy of type II fibers (ActRIIB) in denervated muscles. Therefore, the purpose of the present study was to determine whether PEMFs can alleviate streptozotocin (STZ)-induced diabetic muscle atrophy. To do this, 40 Sprague-Dawley (SD) rats were randomly divided into four groups ( $n=10$ per group): The normal control group (NC; nondiabetic rats without treatment); the diabetic mellitus group (DM; STZ-induced rats without treatment); the diabetic insulin-treated group (DT; diabetic rats on insulin treatment, 6-8 U/d twice a day for 6 weeks) as a positive control; and the diabetic PEMFs therapy group (DP; diabetic rats with PEMFs exposure treatment, $15 \mathrm{~Hz}, 1.46 \mathrm{mT}, 30 \mathrm{~min} /$ day for 6 weeks). Body weight, muscle strength, muscle mass and serum insulin level were significantly increased in the DP group compared with the DM group. PEMFs also decreased the blood glucose level and altered the activity of metabolic enzymes. PEMFs significantly increased the cross-sectional area of muscle fiber. In addition, PEMFs significantly activated protein kinase B (Akt) and mammalian target of rapamycin (mTOR), and inhibited the activity of myostatin (MSTN), ActRIIB and forkhead box protein $\mathrm{O} 1$ (FoxO1) compared with the DM group. Thus indicating that the Akt/mTOR and Akt/FoxO1 signaling pathways may be involved in the promotion of STZ-induced diabetic muscle atrophy by PEMFs. The results of the present
\end{abstract}

Correspondence to: Professor Liang Tang, Institute of Sports Biology, Shaanxi Normal University, 620 West Chang'an Avenue, Chang'an, Xi'an, Shaanxi 710119, P.R. China

E-mail: t1531@snnu.edu.cn

Professor Shucheng An, College of Life Sciences, Shaanxi Normal University, 620 West Chang'an Avenue, Chang'an, Xi'an, Shaanxi 710119, P.R. China

E-mail: shuchengan@snnu.edu.cn

Key words: pulsed electromagnetic fields, diabetes, muscle atrophy, myostatin study suggested that PEMFs stimulation may alleviate diabetic muscle atrophy in the STZ model, and that this is associated with alterations in multiple signaling pathways in which MSTN may be an integral factor. MSTN-associated signaling pathways may provide therapeutic targets to attenuate severe diabetic muscle wasting.

\section{Introduction}

Type 1 diabetes mellitus (T1DM) is an autoimmune disease caused by the destruction of pancreatic $\beta$ cells (1). It affects more than 35 million people worldwide. Reduced muscle mass and myofiber size as well as poor metabolic control in T1DM can result from impaired muscle growth and development (2-4). Diabetic muscle atrophy, a clinical condition, means that the size and strength of skeletal muscles are reduced $(5,6)$, affecting normal daily activities. Protein synthesis or protein degradation is one of the important reasons for diabetic muscle atrophy. Some signaling pathways-such as the activated protein kinase $\mathrm{B}$ (Akt)/ rapamycin (mTOR) and Akt/ forkhead box protein O1 (FoxO1) pathways-may be linked to muscle loss in diabetic muscle atrophy $(7,8)$. Myostatin (MSTN), a member of the transforming growth factor $\beta$ family, is a negative regulator of skeletal muscle growth (9). The negative effect of MSTN may occur via activation of the PI3K/Akt signaling pathway (10).

Growing evidence suggests that pulsed electromagnetic fields (PEMFs) can serve as safe alternatives to drug-based therapies for the treatment of some diseases. In recent years, PEMFs have been widely used in the treatment of osteoporosis, fracture, and other conditions and have produced good therapeutic results. It has been reported, for example, that the atrophy of type II fibers in denervated muscle was retarded by magnetic stimulation (11). However, whether PEMFs can alleviate streptozotocin (STZ)-induced diabetic muscle atrophy has not been investigated.

Accordingly, we examined the effects of PEMFs on STZ-induced diabetic muscle atrophy by evaluating muscle strength, mass, and cross-sectional area of muscle fiber. Furthermore, possible molecular mechanisms were explored through analyses of the gene and protein expression of MSTN, Akt, activin type II receptor (ActRIIB), mTOR, and FoxO1. 


\section{Materials and methods}

Animals. This study was conducted with the approval of the ethics committee of Shaanxi Normal University in Shaanxi, China, and was performed in accordance with the Guide for the Care and Use of Laboratory Animals published by the U.S. National Institutes of Health (NIH; publication no. 85-23, revised 1996).

Healthy male Sprague-Dawley (SD) rats $(200 \pm 20$ g) were obtained from the Laboratory Animal Breeding and Research Center of Xi'an Jiaotong University (Xi'an, China). They were housed in a temperature and humidity controlled room $\left(22 \pm 2^{\circ} \mathrm{C}, 60 \pm 5 \%\right.$ humidity and 12 -hour light/dark cycle). After 5 days of acclimation, the rats were randomly divided into a normal control group (NC; $\mathrm{n}=10)$ and a T1DM model group $(\mathrm{n}=50)$. Experimental T1DM was induced via a peritoneal injection of STZ (Sigma, St. Louis, MO, USA) and $60 \mathrm{mg} / \mathrm{kg}$, $0.1 \mathrm{~mol} / 1$ sodium citrate buffer, $\mathrm{pH}=4.5$ ). An equal volume of buffer was injected into the control rats at the same time. The blood glucose levels in tail vein blood samples were measured on the 1st, 3rd, 7th, and 10th days following injection. The rats with blood glucose levels greater or equal to $16.7 \mathrm{mmol} / \mathrm{l}$ $(300 \mathrm{mg} / \mathrm{dl})$ were considered diabetic. The diabetic rats were then randomly assigned to the DM group $(n=10)$, the diabetic insulin-treated group (DT; n=10) as a positive control, and the diabetic PEMFs therapy group (DP, $\mathrm{n}=10$ ). The DT group was treated with insulin (6-8 U/d twice a day for 6 weeks; Sigma), and the DP group was exposed to PEMFs $(15 \mathrm{~Hz}, 1.46 \mathrm{mT}$, $30 \mathrm{~min} / \mathrm{d}$ for 6 weeks).

PEMFs treatment. The PEMFs exposure system was composed of coils and a pulsed signal generator. There were three identical coils $800 \mathrm{~mm}$ in diameter connected in series and placed coaxially $190 \mathrm{~mm}$ apart. Each coil was made up of enameled coated copper wire $0.8 \mathrm{~mm}$ in diameter. The number of turns on the central coil was 266 , and the number of turns on the two outside coils was 500. The pulsed signal apparatus generated an open circuit waveform composed of a pulsed burst (burst width, $5.16 \mathrm{~ms}$; burst wait, $61.4 \mathrm{~ms}$; pulse width, $0.171 \mathrm{~ms}$; pulse wait, $0.171 \mathrm{~ms}$ ) repeated at $15.08 \mathrm{~Hz}$. The rats in the DP group were put in the center of coils.

Oral glucose tolerance tests. Oral glucose load was administered at $2 \mathrm{~g} / \mathrm{kg}$ of body weight after overnight fasting. Glucose levels were measured from tail bleeds by cutting off a small part of the tail at $0,30,60,90$, and 120 min after glucose administration.

Grip strength. During the final week, forelimb grip strength was measured as maximum tensile force using a rat grip strength meter (YLS-13A; Huaibei Zhenghua Bioinstrumentation Co., Ltd., Anhui, China). Rats were tested 3 times in succession without rest and the results of the 3 tests were averaged for each rat.

Weight and sample preparation. After 6 weeks of treatment, the rats were euthanized with an overdose of diethyl ether and the final body weights were recorded. Blood was collected and centrifuged in order to obtain the serum fractions. Serum was stored at $-80^{\circ} \mathrm{C}$ for further analysis. After the animals were sacrificed, each quadriceps femoris was harvested and weighed, then immediately stored in liquid nitrogen at $-80^{\circ} \mathrm{C}$ for reverse transcription-polymerase chain reaction and western blot analysis.

Biochemical analysis. Blood glucose was measured using the eBsensor Blood Glucose Monitor (Visgeneer Inc., Hsinchu, Taiwan). Serum insulin levels were measured using a commercial enzyme linked immunosorbent assay (ELISA; EMT Millipore, Billerica, MA, USA). The activity levels of succinate dehydrogenase $(\mathrm{SDH})$ and malate dehydrogenase $(\mathrm{MDH})$ in the quadriceps femoris were analyzed with standard colorimetric tests using commercial kits (Nanjing Jiancheng Bioengineering Institute, Nanjing, China) according to the protocols provided by the manufacturer.

Hematoxylin and eosin staining. The quadriceps femoris was cut into $1 \mathrm{mmx} 1 \mathrm{mmx} 1 \mathrm{~mm}$ size and then placed in $2.5 \%$ glutaraldehyde to fix for $24 \mathrm{~h}$ at $4^{\circ} \mathrm{C}$. After washing, the specimens suffered the processes of dehydration and transparency, then were embedded into paraffin and cut into $50 \mathrm{~nm}$ thickness. Subsequently, the paraffin sections undergo the processes of deparaffinage and dehydration, then were stained with hematoxylin for $8 \mathrm{~min}$. After washing, sections were placed into the eosin counterstain for $5 \mathrm{~min}$. After resinene mount, sections were visualized under the optical microscope (Olympus Corp., Tokyo, Japan).

Western blot analysis. The removed quadriceps femoris muscles were homogenized in ice-cold lysis buffer. Protein concentrations were quantified with the BCA protein assay kit (Thermo Fisher Scientific, Inc., Waltham, MA, USA). Protein concentrations were determined and equal amounts of sample were uploaded for sodium dodecyl sulfate polyacrylamide gel electrophoresis (SDS-PAGE) analysis. After electrophoresis and separation, samples were transferred onto nitrocellulose membranes. The immunoblots were incubated with primary antibodies overnight at $4^{\circ} \mathrm{C}$, followed by incubation with the corresponding secondary antibodies at room temperature for $1 \mathrm{~h}$. Blots were visualized with ECL-plus reagent, and the results were quantified with Lab Image Version 2.7.1. The primary antibodies used were as follows: the expression of MSTN [EPR4567(2), ab124721], ActRIIB (EPR10739, ab180185) from Abcam (Cambridge, UK); AKT (Rabbit Ab 9272S); phospho-AKT (S473 9271S); mTOR (Rabbit 2972S), phospho-mTOR (S2448, 2971P), FoxO1 (C29H4, Rabbit 2880P), and phospho-FoxO1 (S256, 9461P) from Cell Signaling Technology, Inc. (Danvers, MA, USA).

Statistical analysis. Statistical analysis was performed using SPSS version 20.0 software (IBM Corp., Armonk, NY, USA). One-way analysis of variance was employed to evaluate the differences between the three groups. Once a significant difference was detected, Tukey's multiple comparisons test was used to determine the significance between any two groups. $\mathrm{P}<0.05$ was considered to indicate a statistically significant difference. The results are expressed as the mean \pm standard deviation.

\section{Results}

Changes of blood glucose and oral glucose tolerance tests before and after model establishment. Blood glucose levels 

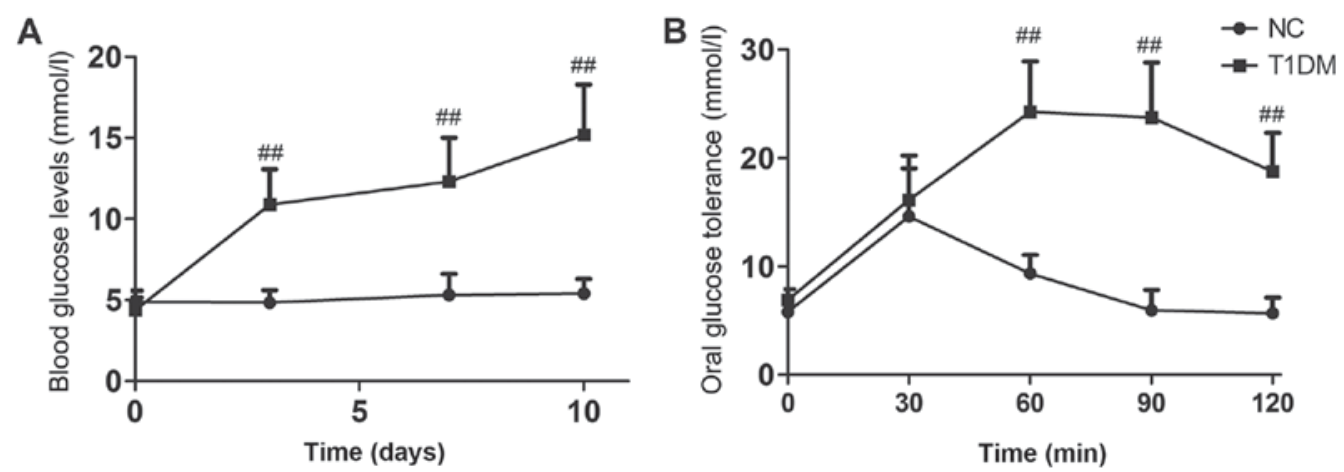

Figure 1. Comparison of (A) blood glucose levels and (B) oral glucose tolerance in the NC and T1DM groups prior to and following model establishment. Data are expressed as mean \pm standard deviation ( $\mathrm{n}=8$ /group). ${ }^{\# \#} \mathrm{P}<0.01$ vs. NC. T1DM, type 1 diabetes mellitus; NC, normal control.
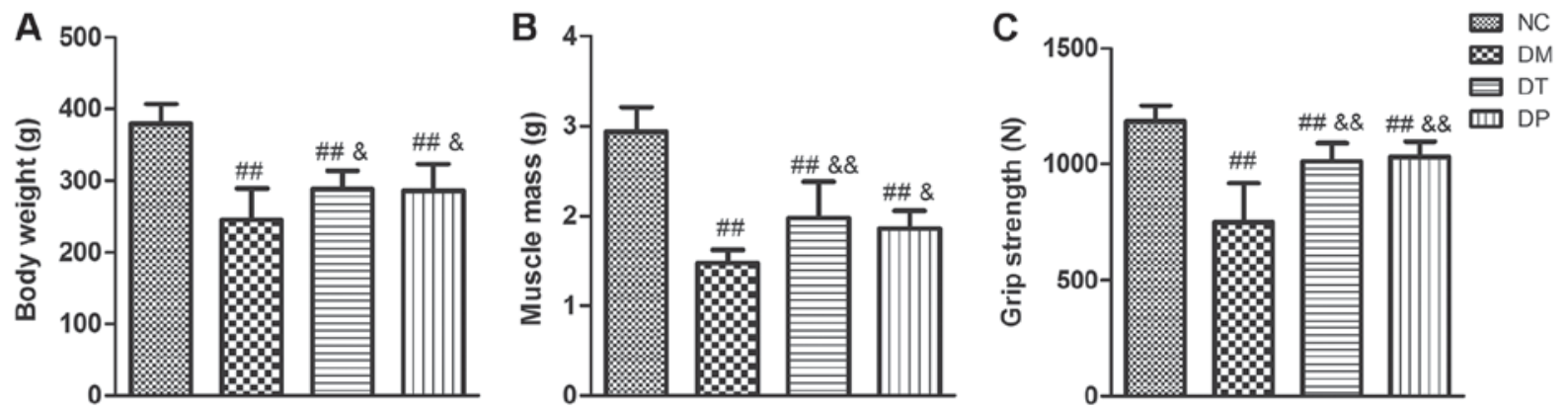

Figure 2. Effects of pulsed electromagnetic fields on (A) serum body weight, (B) muscle weight and (C) muscle strength. Data are expressed as the mean \pm standard deviation ( $\mathrm{n}=8 /$ group). ${ }^{\# \#} \mathrm{P}<0.01$ vs. NC; ${ }^{\&} \mathrm{P}<0.05$ and ${ }^{\& \&} \mathrm{P}<0.01$ vs. DM. NC, normal control; DM, diabetic mellitus group; DT, diabetic insulin-treated group; DP, diabetic pulsed electromagnetic fields-therapy group.

in diabetic mice remained extremely high throughout the experiment $(\mathrm{P}<0.01$, Fig. 1A). Oral glucose tolerance tests were performed after 6 weeks of treatment. Rats in the DB group showed impaired glucose tolerance compared with those in the NC group ( $\mathrm{P}<0.01$, Fig. 1B). The T1DM model was successfully established.

PEMFs increased body weight, muscle weight and muscle strength. After 6 weeks of PEMFs treatment, the body weight, quadriceps wet weight, and grip strength of the rats were measured (as shown in Fig. 2). Compared with the NC group, the body weight, quadriceps wet weight, and grip strength of the $\mathrm{DM}$ group were significantly decreased $(\mathrm{P}<0.01, \mathrm{P}<0.01$, and $\mathrm{P}<0.01$, respectively). Although the body weight, muscle weight, and grip strength in the DT and DF groups were significantly lower than those in the $\mathrm{NC}$ group $(\mathrm{P}<0.01, \mathrm{P}<0.01$, and $\mathrm{P}<0.01$, respectively), insulin treatment statistically significantly affected the loss of body weight, muscle weight, and grip strength $(\mathrm{P}<0.01, \mathrm{P}<0.01$, and $\mathrm{P}<0.01$, respectively) in this group compared with the DM group. Furthermore, the body weight, muscle weight, and grip strength in the DF group were significantly increased $(\mathrm{P}<0.05, \mathrm{P}<0.05$, and $\mathrm{P}<0.01$, respectively) compared with the $\mathrm{DM}$ group.

PEMFs decreased blood glucose level and increased insulin level. Before the animals were sacrificed, the blood glucose levels were detected. The blood glucose levels of the DM group were significantly higher than those of the NC group $(\mathrm{P}<0.01)$; insulin and $\mathrm{PEMFs}$ treatment caused a decrease in blood glucose levels compared with the DM group $(\mathrm{P}<0.01)$ (Fig. 3A). Moreover, insulin levels were significantly decreased in the DM group $(\mathrm{P}<0.01)$. Insulin and PEMFs treatment caused a significant decrease in blood glucose $(\mathrm{P}<0.01$ and $\mathrm{P}<0.01$, respectively) and a significant increase in serum insulin compared with the DM group $(\mathrm{P}<0.01$ and $\mathrm{P}<0.05$, respectively) (Fig. 3B).

PEMFs altered the activities of metabolic enzymes. Fig. 4 shows the effect of PEMFs on activity levels of key muscle metabolism enzymes in the several experimental groups. In the diabetic rats, activity levels of SDH (Fig. 4A) and $\mathrm{MDH}$ (Fig. 4B) were significantly decreased $(\mathrm{P}<0.01$ and $\mathrm{P}<0.01$, respectively) as compared with the normal, nondiabetic rats. Insulin treatment statistically significantly increased the SDH and $\mathrm{MDH}$ levels $(\mathrm{P}<0.05$ and $\mathrm{P}<0.05$, respectively). PEMFs treatment resulted in significantly higher SDH and $\mathrm{MDH}$ activity levels $(\mathrm{P}<0.05$ and $\mathrm{P}<0.05$, respectively) as compared with the untreated diabetic rats.

Effect of PEMFs on cross-sectional area of muscle fiber. As shown in Fig. 5, the cross-sectional area of muscle fiber in DM group was significantly decreased $(\mathrm{P}<0.01)$ as compared with the normal. The cross-sectional area of muscle fiber in DT and DP groups were significantly increased $(\mathrm{P}<0.01$ and $\mathrm{P}<0.05$, respectively) as compared with the DM group.

Effect of PEMFs on MSTN/Akt/mTOR/FoxO signaling pathways. To investigate the molecular mechanism of the effect of PEMFs 

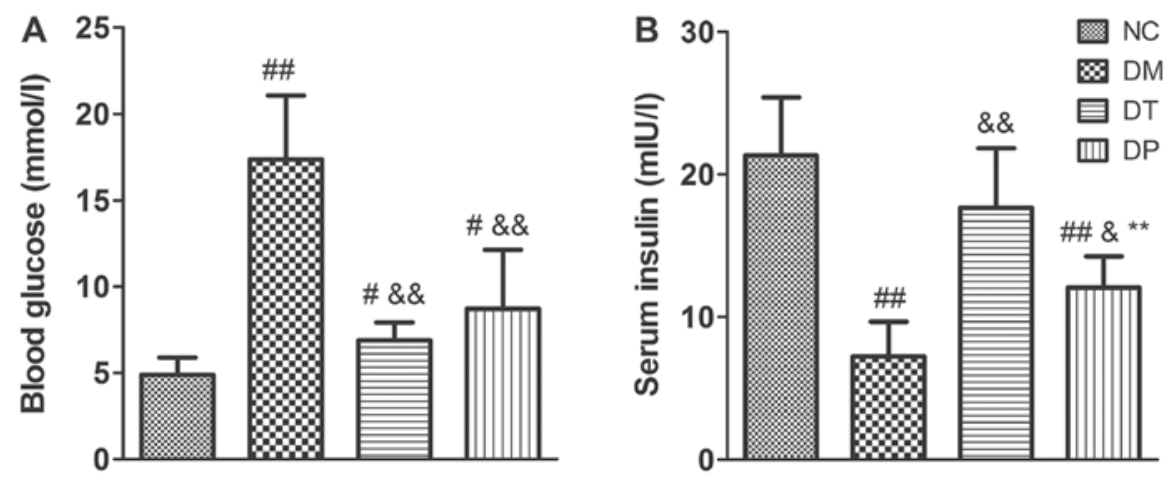

Figure 3. Effects of pulsed electromagnetic fields on (A) blood glucose level and (B) increased insulin level. Data are expressed as the mean \pm standard deviation ( $\mathrm{n}=8$ /group). ${ }^{\#} \mathrm{P}<0.05$ and ${ }^{\# \#} \mathrm{P}<0.01$ vs. $\mathrm{NC} ;{ }^{\&} \mathrm{P}<0.05$ and ${ }^{\& \&} \mathrm{P}<0.01$ vs. $\mathrm{DM} ;{ }^{* *} \mathrm{P}<0.01$ vs. DT. NC, normal control; DM, diabetic mellitus group; DT, diabetic insulin-treated group; DP, diabetic pulsed electromagnetic fields-therapy group.
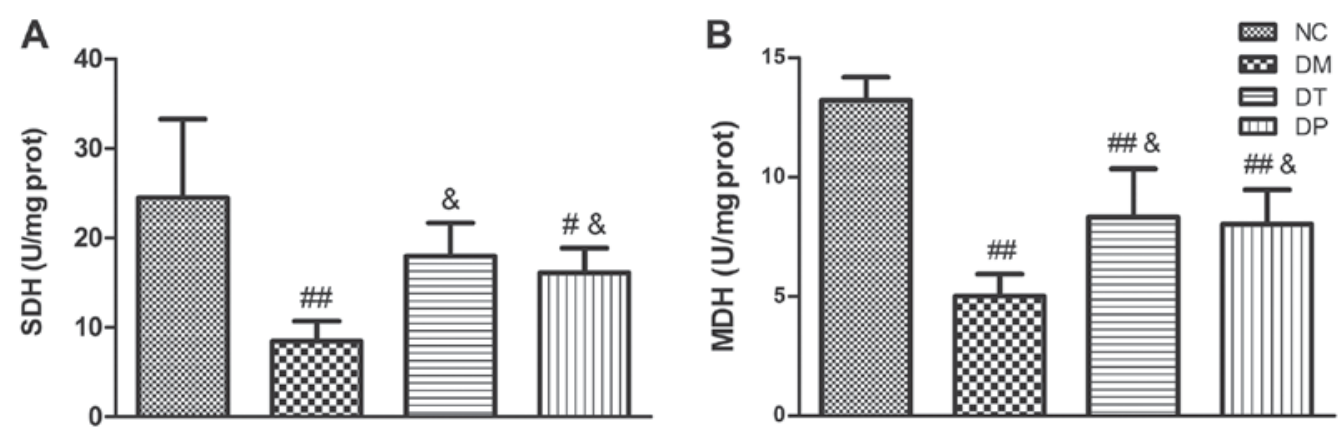

Figure 4. Effects of pulsed electromagnetic fields on the levels of (A) SDH and (B) MDH. Data are expressed as the mean \pm standard deviation (n=8/group) ${ }^{\#} \mathrm{P}<0.05$ and ${ }^{\# \#} \mathrm{P}<0.01$ vs. NC; ${ }^{\mathrm{P}}<0.05$ vs. DM. SDH, succinate dehydrogenase; $\mathrm{MDH}$, malate dehydrogenase; $\mathrm{NC}$, normal control; DM, diabetic mellitus group; DT, diabetic insulin-treated group; DP, diabetic pulsed electromagnetic fields-therapy group.

on reversal of muscle atrophy in diabetic rats, we explored the MSTN, Akt/mTOR, and FoxO3 signaling pathways, which are closely related to muscle protein synthesis and subject to degradation by Western blotting. The results showed that STZ markedly decreased p-Akt $(\mathrm{P}<0.01)$ (Fig. 6D), mTOR $(\mathrm{P}<0.01)$ (Fig. 6E), and p-mTOR $(\mathrm{P}<0.01)$ (Fig. 6F) while it increased MSTN $(\mathrm{P}<0.01)$ (Fig. 6A), ActRIIB ( $\mathrm{P}<0.01)$ (Fig. 6B), FoxO1 $(\mathrm{P}<0.01)$ (Fig. 6G), and p-FoxO1 $(\mathrm{P}<0.01)$ (Fig. 6H) as compared with the nondiabetic rats. Insulin and PEMF increased p-Akt $(\mathrm{P}<0.01$ and $\mathrm{P}<0.05$, respectively) (Fig. 6D), $\mathrm{mTOR}(\mathrm{P}<0.01$ and $\mathrm{P}<0.01$, respectively) (Fig. 6E), p-mTOR $(\mathrm{P}<.01$ and $\mathrm{P}<0.01$, respectively) (Fig. 6F) whereas it reduced MSTN $(\mathrm{P}<0.01)$ (Fig. 6A), ActRIIB ( $\mathrm{P}<0.01$ and $\mathrm{P}<0.01$, respectively) (Fig. 6B), FoxO1 ( $\mathrm{P}<0.01$ and $\mathrm{P}<0.05$, respectively) (Fig. 6G), and $\mathrm{p}$-FoxO1 $(\mathrm{P}<0.01$ and $\mathrm{P}<0.01$, respectively) (Fig. $6 \mathrm{H})$ as compared with the DM group. Akt expression did not change significantly among the groups (Fig. 6C). These results suggest that PEMFs can not only increase muscle protein synthesis by activating Akt/mTOR but also inhibit protein degradation by inactivating MSTN and FoxO1 protein.

\section{Discussion}

Because of the absence of insulin in T1DM, blood glucose levels rise dramatically when glucose cannot be taken up into the major insulin-sensitive tissue-skeletal muscle.

Individuals with T1DM are at a high risk of muscle atrophy. This means that many of these individuals' lives are lost, despite insulin therapies (12). PEMFs are dynamic and able to penetrate all the way through the body, thus having many effects. It has been proved that PEMFs can promote the proliferation and differentiation of $\mathrm{C} 2 \mathrm{C} 12$ myoblasts $(13,14)$ and facilitate tendon healing (15). Therefore, we sought to investigate the effects and potential mechanisms of PEMFs on STZ-induced diabetic muscle atrophy. Our findings indicate that PEMFs alleviate diabetic myopathy by increasing protein synthesis and decreasing protein degradation in MSTN-associated signaling pathways.

Increase in blood glucose, appetite, urination, and thirst during the experimental period confirmed the induction of T1DM. The insufficient insulin therapy could cause the development of muscle function in individuals with T1DM. Furthermore, glycemic control is directly related to muscle metabolism and could be an important determinant of muscle force and power in T1DM (16). In accordance with earlier studies (17-19), our experimental rats with T1DM were characterized by increased blood glucose, appetite, urination, and thirst and by decreased blood insulin, weight mass, muscle mass, and grip strength compared with the NC group rats. Furthermore, compared with DM, PEMFs significantly increased strength and mass of quadriceps muscle, and cross-sectional area of quadriceps muscle fiber. These results indicate that PEMFs can improve the muscle atrophy induced by STZ.

It is well known that SDH and MDH are marker enzymes in the metabolism of muscle $(20,21)$. Decreased SDH and MDH activity in patients with diabetes has been reported (22-25). 

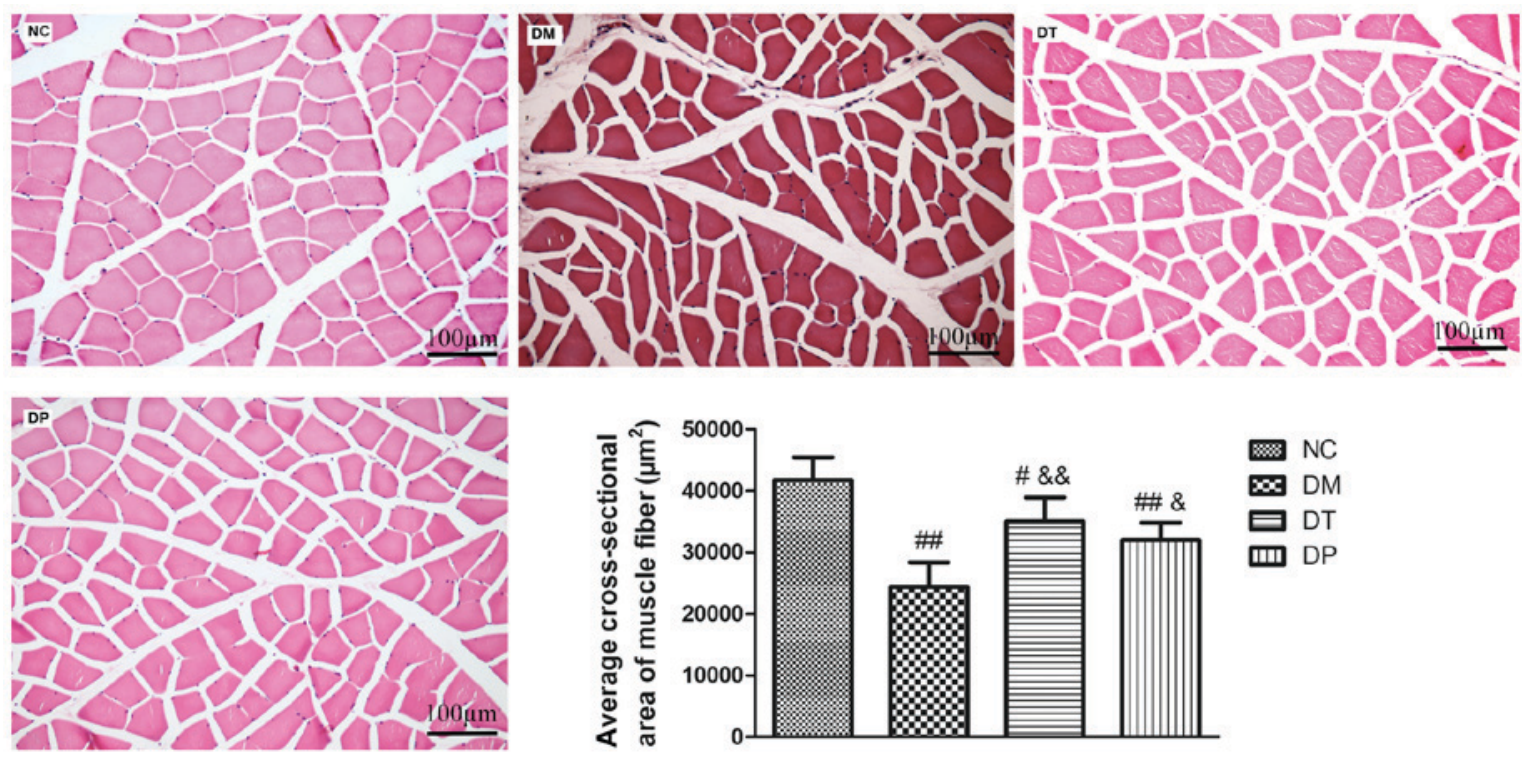

Figure 5. Effect of pulsed electromagnetic fields on cross-sectional area of muscle fiber. Data are expressed the mean \pm standard deviation (n=8/group). Each sample represents ten different fields of view, images were taken at magnification x 200 under a light microscope (scale bars, $100 \mu \mathrm{m}$ ). ${ }^{\#} \mathrm{P}<0.05$ and ${ }^{\prime \prime \prime} \mathrm{P}<0.01 \mathrm{vs}$. NC; ${ }^{\text {\& }} \mathrm{P}<0.05$ and ${ }^{\&}$ P $<0.01$ vs. DM. NC, normal control; DM, diabetic mellitus group; DT, diabetic insulin-treated group; DP, diabetic pulsed electromagnetic fields-therapy group.

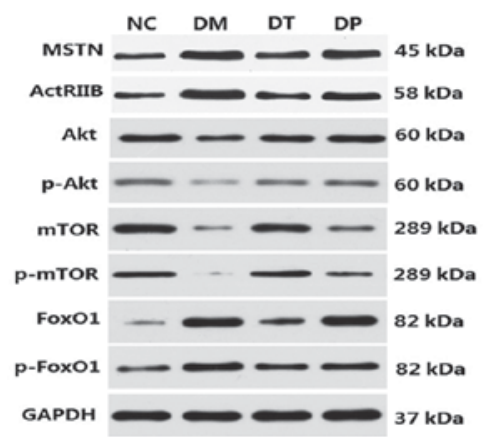

A
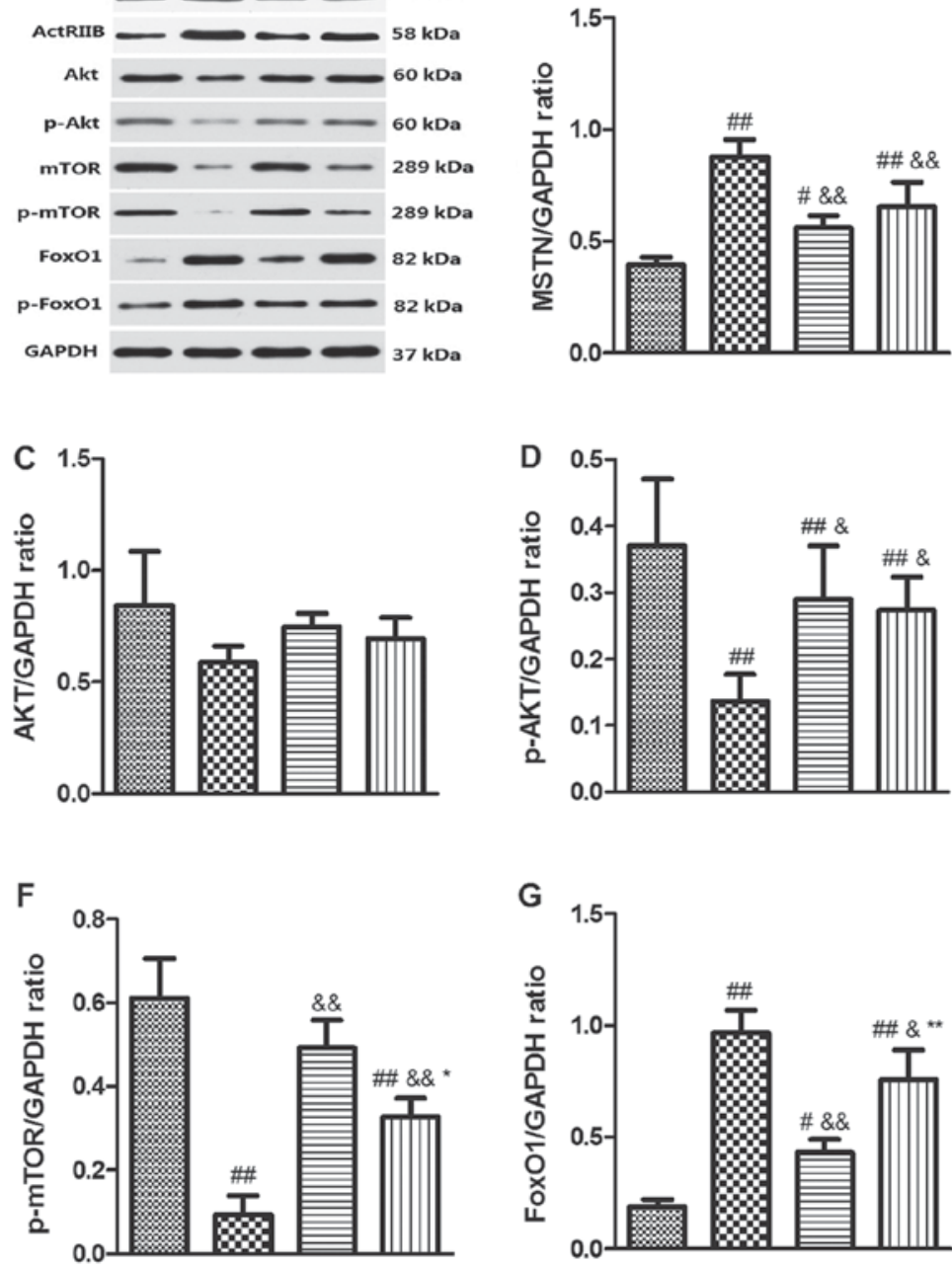
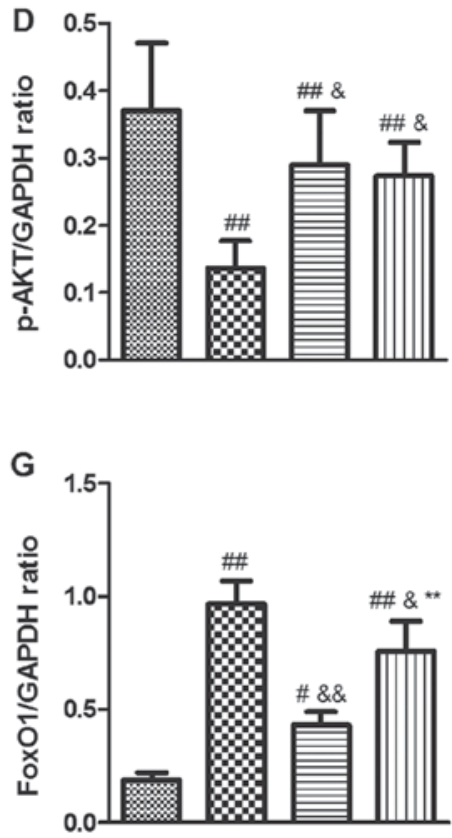

B
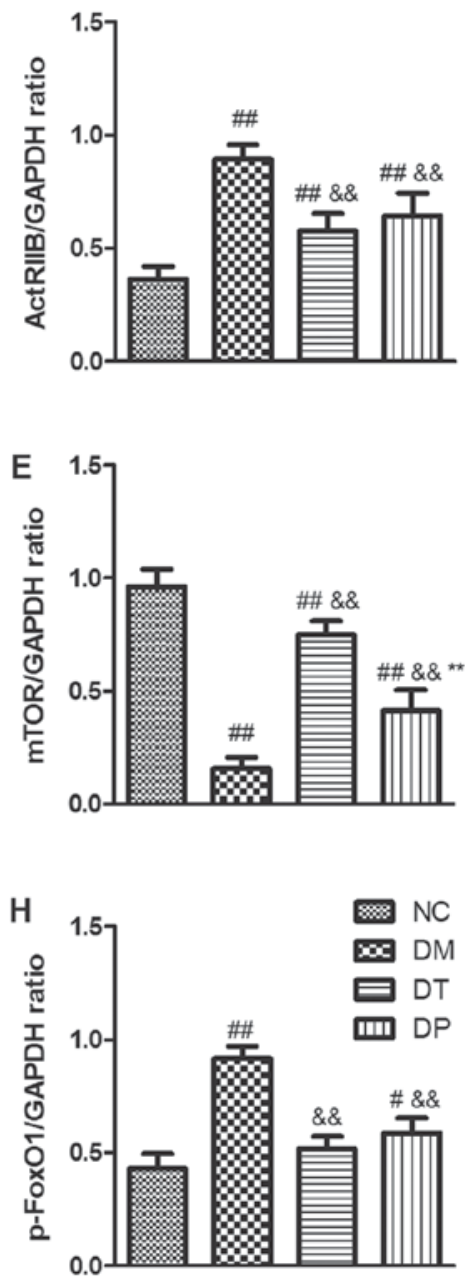

Figure 6. Effects of pulsed electromagnetic fields on the protein expressions of (A) MSTN, (B) ActRIIB, (C) Akt, (D) p-Akt, (E) mTOR, (F) p-mTOR, (G) FoxO1 and $(\mathrm{H}) \mathrm{p}$-FoxO1 in quadriceps. Data are expressed as the mean \pm standard deviation. ${ }^{\#} \mathrm{P}<0.05$ and ${ }^{\# \prime \prime} \mathrm{P}<0.01$ vs. NC; ${ }^{\circledR} \mathrm{P}<0.05$ and ${ }^{\& \&} \mathrm{P}<0.01$ vs. DM; ${ }^{\mathrm{P}}<0.05$ and ${ }^{* *} \mathrm{P}<0.01$ vs. DT. NC, normal control; DM, diabetic mellitus group; DT, diabetic insulin-treated group; DP, diabetic pulsed electromagnetic fields-therapy group; MSTN, myostatin; ActRIIB, activin type II receptor; Akt, protein kinase B; mTOR, mammalian target of rapamycin; p-, phosphorylated; FoxO1, forkhead box protein $\mathrm{O} 1$ 
In the current study, SDH and MDH activity was decreased in the muscles of diabetic rats. These results are in line with previous studies. The observed increase in the activity of SDH and $\mathrm{MDH}$ in the quadriceps femoris muscles of the diabetic rats was significantly enhanced by PEMFs therapy, indicating that PEMFs contributed to increasing the metabolic capacity of skeletal muscle by alleviating STZ-induced diabetic muscle atrophy.

MSTN is a potent negative regulator of skeletal muscle mass as demonstrated by the hypermuscularity caused by its inactivation (26). MSTN is expressed in skeletal muscle predominantly, and the muscle mass increases significantly if its gene is disrupted (9). It has been demonstrated that MSTN can activate the TGF $\beta 2$ /activin type II receptor (ActRIIB) and then activate the downstream signaling pathway (27,28). Animals with STZ-induced T1DM that were treated with follistatin (an inhibitor of MSTN) demonstrated improvement in the regenerative capacity of skeletal muscle (29), and elevations in MSTN expression have been observed in STZ-induced T1DM $(7,30)$. In accordance with earlier studies, our results also pointed to a significant increase in MSTN expression in the animals with STZ-induced T1DM. PEMFs significantly inhibited mRNA and protein expression of MSTN and ActRIIB compared with DM. These results indicate that inhibition of MSTN may play a role in PEMFs promotion of STZ-induced diabetic muscle atrophy.

Muscle mass depends on a homeostatic balance between protein synthesis and degradation. It is believed that the Akt-mTOR pathway is the principal signaling protein cascade regulating protein synthesis $(31,32)$. Akt is a key regulator of several signaling pathways associated with skeletal muscle homeostasis, and its major direct target downstream is the mammalian target of mTOR kinase (33). The mTOR blocker can inhibit muscle hypertrophy (34). Furthermore, inhibition of the Akt/mTOR pathway can lead to muscle atrophy (35). Rodriguez et al (36) have reported that MSTN negatively regulates the activity of the Akt pathway, which promotes protein synthesis.

Moreover, muscle-specific FoxO1 overexpression in mice has been linked to muscle atrophy (37), and Akt can control the activation of FoxO transcription factors (38). In the present study, STZ enhanced the activity of Akt and mTOR and inhibited FoxO1 activity and then induced skeletal muscle hypertrophy $(7,39)$. Some findings suggest that PEMFs exposure might function in a manner analogous to soluble growth factors by activating a unique set of signaling pathways, including the Akt/mTOR pathway (40). We found that PEMFs significantly activated Akt and mTOR and inhibited the activity of MSTN, ActRIIB, and FoxO1 compared with DM. This means that both the Akt/mTOR and Akt/FoxO1 signaling pathways may be involved in the alleviation of STZ-induced diabetic muscle atrophy by PEMFs.

In terms of broadness, future studies should investigate the mechanistic links between the proteins identified in our study and muscle atrophy in various diabetic models and investigate methods of inhibiting or knocking down elements of the MSTN pathway. In terms of depth, changes in vascular lesions and nerve evoked potentials as a more in-depth discussion of electromagnetic field effects on diabetic muscle atrophy, which is the focus of our next study. We will be ready to cooperate with electrophysiology laboratories to research this issue.
Our results show that PEMFs stimulation can alleviate diabetic muscle atrophy in an STZ model in association with the alteration of multiple signaling pathways, wherein MSTN may be an important factor. MSTN-associated signaling pathways may provide therapeutic targets for the attenuation of severe diabetic muscle wasting.

\section{Acknowledgements}

The authors would like to thank the graduate students of the Institute of Sports Biology, Shaanxi Normal University (Shaanxi, China) for their cooperation, and the College of Life Sciences, Shaanxi Normal University, and Department of Physical Education, Xi'an University of Post and Telecommunications (Shaanxi, China).

\section{Funding}

The present study was supported by the National Natural Science Foundation of China (grant nos. 11774213, 11727813 and 11502134).

\section{Availability of data and materials}

The datasets used and/or analyzed during the current study are available from the corresponding author on reasonable request.

\section{Authors' contributions}

LT and SA conceived the study. JY, BY, XF, LS and YK performed the experiments, and collected and analyzed the data. JY and LS prepared the manuscript. XF revised the manuscript and all authors edited the manuscript. All authors contributed to the writing of the manuscript.

\section{Ethics approval and consent to participate}

The present study was conducted with the approval of the Ethics Committee of Shaanxi Normal University (Shaanxi, China).

\section{Consent for publication}

Not applicable.

\section{Competing interests}

The authors declare that they have no competing interests.

\section{References}

1. Atkinson MA and Eisenbarth GS: Type 1 diabetes: New perspectives on disease pathogenesis and treatment. Lancet 358: 221-229, 2001.

2. Andersen H, Gjerstad MD and Jakobsen J: Atrophy of foot muscles: A measure of diabetic neuropathy. Diabetes Care 27: 2382-2385, 2004.

3. Fritzsche K, Blüher M, Schering S, Buchwalow IB, Kern M, Linke A, Oberbach A, Adams V and Punkt K: Metabolic profile and nitric oxide synthase expression of skeletal muscle fibers are altered in patients with type 1 diabetes. Exp Clin Endocrinol Diabetes 116: 606-613, 2008. 
4. Krause MP, Riddell MC, Gordon CS, Imam SA, Cafarelli E and Hawke TJ: Diabetic myopathy differs between Ins2Akita+/- and streptozotocin-induced Type 1 diabetic models. J Appl Physiol (1985) 106: 1650-1659, 2009

5. Jakobsen J and Reske-Nielsen E: Diffuse muscle fiber atrophy in newly diagnosed diabetes. Clin Neuropathol 5: 73-77, 1986.

6. Riddell MC and Iscoe KE: Physical activity, sport, and pediatric diabetes. Pediatr Diabetes 7: 60-70, 2006.

7. Hulmi JJ, Silvennoinen M, Lehti M, Kivelä R and Kainulainen $\mathrm{H}$ : Altered REDD1, myostatin, and Akt/mTOR/FoxO/MAPK signaling in streptozotocin-induced diabetic muscle atrophy. Am J Physiol Endocrinol Metab 302: E307-E315, 2012.

8. Tang L, Liu CT, Wang XD, Luo K, Zhang DD, Chi AP, Zhang J and Sun LJ: A prepared anti-MSTN polyclonal antibody reverses insulin resistance of diet-induced obese rats via regulation of $\mathrm{PI} 3 \mathrm{~K} / \mathrm{Akt} / \mathrm{mTOR} \&$ FoxO1 signal pathways. Biotechnol Lett 36: 2417-2423, 2014.

9. McPherron AC, Lawler AM and Lee SJ: Regulation of skeletal muscle mass in mice by a new TGF-beta superfamily member Nature 387: 83-90, 1997.

10. Glass DJ: PI3 kinase regulation of skeletal muscle hypertrophy and atrophy. Curr Top Microbiol Immunol 346: 267-278, 2010.

11. Chang CW and Lien IN: Tardy effect of neurogenic muscular atrophy by magnetic stimulation. Am J Phys Med Rehabil 73: 275-279, 1994.

12. Livingstone SJ, Levin D, Looker HC, Lindsay RS, Wild SH, Joss N, Leese G, Leslie P, McCrimmon RJ, Metcalfe W, et al: Estimated life expectancy in a Scottish cohort with type 1 diabetes, 2008-2010. JAMA 313: 37-44, 2015.

13. Xu H, Zhang J, Lei Y, Han Z, Rong D, Yu Q, Zhao M and Tian J: Low frequency pulsed electromagnetic field promotes C2C12 myoblasts proliferation via activation of MAPK/ERK pathway. Biochem Biophys Res Commun 479: 97-102, 2016.

14. Liu M, Lee C, Laron D, Zhang N, Waldorff EI, Ryaby JT, Feeley B and Liu X: Role of pulsed electromagnetic fields (PEMF) on tenocytes and myoblasts-potential application for treating rotator cuff tears. J Orthop Res 35: 956-964, 2017

15. Tucker JJ, Cirone JM, Morris TR, Nuss CA, Huegel J, Waldorff EI, Zhang N, Ryaby JT and Soslowsky LJ: Pulsed electromagnetic field therapy improves tendon-to-bone healing in a rat rotator cuff repair model. J Orthop Res 35: 902-909, 2017.

16. Fricke O, Seewi O, Semler O, Tutlewski B, Stabrey A and Schoenau E: The influence of auxology and long-term glycemic control on muscle function in children and adolescents with type 1 diabetes mellitus. J Musculoskelet Neuronal Interact 8: $188-195,2008$

17. Junod A, Lambert AE, Stauffacher W and Renold AE: Diabetogenic action of streptozotocin: Relationship of dose to metabolic response. J Clin Invest 48: 2129-2139, 1969.

18. Li RJ, Qiu SD, Tian H and Zhou SW: Diabetes induced by multiple low doses of STZ can be spontaneously recovered in adult mice. Dongwuxue Yanjiu 34: 238-243, 2013 (In Chinese).

19. Tsai CC, Chan P, Chen LJ, Chang CK, Liu Z and Lin JW: Merit of ginseng in the treatment of heart failure in type 1-like diabetic rats. Biomed Res Int 2014: 484161, 2014.

20. Lewis MI, Fournier M, Wang H, Storer TW, Casaburi R and Kopple JD: Effect of endurance and/or strength training on muscle fiber size, oxidative capacity, and capillarity in hemodialysis patients. J Appl Physiol (1985) 119: 865-871, 2015

21. Eprintsev AT, Falaleeva MI, Lyashchenko MS, Gataullinaa MO and Kompantseva EI: Isoformes of malate dehydrogenase from rhodovulum steppense A-20s grown chemotrophically under aerobic condtions. Prikl Biokhim Mikrobiol 52: 168-173, 2016.

22. Chen V and Ianuzzo CD: Metabolic alterations in skeletal muscle of chronically streptozotocin-diabetic rats. Arch Biochem Biophys 217: 131-138, 1982.
23. Ianuzzo CD and Armstrong RB: Phosphofructokinase and succinate dehydrogenase activities of normal and diabetic rat skeletal muscle. Horm Metab Res 8: 244-245, 1976.

24. Cai F: Studies of enzyme histochemistry and ultrastructure of the myocardium in rats with streptozotocin-induced diabetes. Zhonghua Yi Xue Za Zhi 69: 276-278, 20, 1989 (In Chinese).

25. Jia Q, Ma S, Liu X, Li S, Wang Y, Gao Q and Yang R: Effects of hydrogen sulfide on contraction capacity of diaphragm from type 1 diabetic rats. Zhong Nan Da Xue Xue Bao Yi Xue Ban 41: 496-501, 2016 (In Chinese).

26. Lee SJ: Sprinting without myostatin: A genetic determinant of athletic prowess. Trends Genet 23: 475-477, 2007.

27. Rebbapragada A, Benchabane H, Wrana JL, Celeste AJ and Attisano L: Myostatin signals through a transforming growth factor beta-like signaling pathway to block adipogenesis. Mol Cell Biol 23: 7230-7242, 2003.

28. Lee SJ: Regulation of muscle mass by myostatin. Annu Rev Cell Dev Biol 20: 61-86, 2004

29. Jeong J, Conboy MJ and Conboy IM: Pharmacological inhibition of myostatin/TGF- $\beta$ receptor/pSmad3 signaling rescues muscle regenerative responses in mouse model of type 1 diabetes. Acta Pharmacol Sin 34: 1052-1060, 2013

30. Sriram S, Subramanian S, Juvvuna PK, McFarlane C, Salerno MS, Kambadur R and Sharma M: Myostatin induces DNA damage in skeletal muscle of streptozotocin-induced type 1 diabetic mice. J Biol Chem 289: 5784-5798, 2014.

31. Glass DJ: Skeletal muscle hypertrophy and atrophy signaling pathways. Int J Biochem Cell Biol 37: 1974-1984, 2005.

32. Leger B, Cartoni R, Praz M, Lamon S, Dériaz O, Crettenand A, Gobelet C, Rohmer P, Konzelmann M, Luthi F and Russell AP: Akt signalling through GSK-3beta, mTOR and Foxol is involved in human skeletal muscle hypertrophy and atrophy. J Physiol 576: 923-933, 2006

33. Dang K, Li YZ, Gong LC, Xue W, Wang HP, Goswami N and Gao YF: Stable atrogin-1 (Fbxo32) and MuRF1 (Trim63) gene expression is involved in the protective mechanism in soleus muscle of hibernating Daurian ground squirrels (Spermophilus dauricus). Biol Open 5: 62-71, 2016.

34. Bodine SC, Stitt TN, Gonzalez M, Kline WO, Stover GL, Bauerlein R, Zlotchenko E, Scrimgeour A, Lawrence JC, Glass DJ and Yancopoulos GD: Akt/mTOR pathway is a crucial regulator of skeletal muscle hypertrophy and can prevent muscle atrophy in vivo. Nat Cell Biol 3: 1014-1019, 2001.

35. Hoffman EP and Nader GA: Balancing muscle hypertrophy and atrophy. Nat Med 10: 584-585, 2004.

36. Rodriguez J, Vernus B, Chelh I, Cassar-Malek I, Gabillard JC, Hadj Sassi A, Seiliez I, Picard B and Bonnieu A: Myostatin and the skeletal muscle atrophy and hypertrophy signaling pathways. Cell Mol Life Sci 71: 4361-4371, 2014.

37. Kamei Y, Miura S, Suzuki M, Kai Y, Mizukami J, Taniguchi T, Mochida K, Hata T, Matsuda J, Aburatani H, et al: Skeletal muscle FOXO1 (FKHR) transgenic mice have less skeletal muscle mass, down-regulated Type I (slow twitch/red muscle) fiber genes, and impaired glycemic control. J Biol Chem 279: 41114-41123, 2004.

38. Bonaldo P and Sandri M: Cellular and molecular mechanisms of muscle atrophy. Dis Model Mech 6: 25-39, 2013.

39. Zhang J, Zhuang P, Wang Y, Song L, Zhang M, Lu Z, Zhang L, Wang J, Alemu PN, Zhang Y, et al: Reversal of muscle atrophy by Zhimu-Huangbai herb-pair via Akt/mTOR/FoxO3 signal pathway in streptozotocin-induced diabetic mice. PLoS One 9: e100918, 2014.

40. Patterson TE, Sakai Y, Grabiner MD, Ibiwoye M, Midura RJ, Zborowski M and Wolfman A: Exposure of murine cells to pulsed electromagnetic fields rapidly activates the mTOR signaling pathway. Bioelectromagnetics 27: 535-544, 2006. 